\title{
MEDIATING ROLE OF DISSOCIATION SYMPTOMS BETWEEN ADOLESCENT COMPULSIVE INTERNET USE ACROSS TIME
}

\author{
Anika Miltuze \\ University of Latvia, Latvia \\ Sandra Beatrice Sebre \\ University of Latvia, Latvia
}

\begin{abstract}
The aim of this study was to examine adolescent compulsive internet use (CIU) across a one-year period, to examine associations with symptoms of depression, anxiety and dissociation, as well as to analyze the potential mediating effect of dissociation symptoms. Previous studies have indicated that dissociation might serve as a coping strategy and/or as a consequence of problematic internet use. Participating in the study were 80 adolescents (39 girls and 41 boys), ages from 12 to 18 (mean age 14.90 years) at the time of the first measurement. At Time 1 and one year later at Time 2 measurement points the participants completed the Trauma Symptom Checklist (Briere, 1995) and items from the Compulsive Internet Use Scale (Meerkert, VanDen Eijnden, Vermulst, \& Garretsen, 2009). Correlation analyses showed associations between CIU and the adolescents' ratings of anxiety, depression and dissociation at Time 1 and Time 2. Mediation analysis indicated partial mediation of dissociation symptoms between CIU at Time 1 and Time 2. A comparison of these relationships across time provides opportunity to discuss possible directions of causality and implications of bidirectionality between dissociative tendencies and adolescent prolonged, compulsive internet use.
\end{abstract}

Keywords: adolescents, compulsive internet use, dissociation symptoms.

\section{Introduction}

Although the ubiquitous presence of the internet in the daily lives of modern day adolescents and adults provides undoubtable benefits, there are also potential costs. Mazzoni and colleagues (2017) have suggested that the benefits which internet usage provides include the boosting of human potential and exploration of previously unforeseen possibilities. The rapid access to vast realms of information and the potential of electronically manipulating with this information can help to stimulate intelligence and creativity. Web technologies may serve as a functional tool to be used as a source of empowerment, and benefits of internet usage can also include enhanced social connectedness. Internet-based interventions have been found to be effective in the treatment of 
various psychiatric disorders (e.g. Probst et al., 2020). However, Ekbia and Nardi (2012) suggest that internet usage may at times lead to "inverse instrumentality”, whereby the user may lose control over the tool.

The loss of control over internet usage was initially conceptualized by Young (1998) as Internet Addiction, whereby this type of addiction was compared to other forms such as gambling, drugs and alcohol use because of the similarities in preoccupation and withdrawal symptoms. However, since then various researchers and clinicians have asserted that "internet addiction" may not be the most precise description of this phenomenon because individuals are not addicted to the internet per se, but rather have developed a compulsion to engage in specific forms of internet usage, resulting in subsequent complications in their daily life. Hence, difficulty in controlling one's use of the internet is also referred to as compulsive internet use (CIU) or problematic internet use (PIU), terms which are used as synonyms. CIU or PIU is not a diagnosis, but rather refers to preoccupation with internet use, difficulty in controlling one's use of the internet, the need for increasingly greater time spent using the internet, and withdrawal symptoms such as irritability when one is not using the internet (Meerkerk, Van Den Eijnden, Vermulst, \& Garretsen, 2009).

Previous research has pointed to associations between PIU and psychopathology, most ofen indicating links with depression, anxiety or aggression (Kaess et al., 2014). For example, a review article of 20 studies on internet use reported that $75 \%$ of those studies found significant associations between PIU and depression, 57\% found links between PIU and anxiety disorders, and 66\% connected PIU with hostility or aggression (Carli et al., 2012). However, there have been very few studies examining the relationships between internet use and symptoms of dissociation, with a few exceptions (e.g. Dalbudak, Evren, Aldemir, \& Evren, 2014; De Berardis et al., 2009).

Dissociation has been conceptualized as experiencing a disconnection and lack of continuity between thoughts, memories, surrounding, actions and identity. Dissociative experiences include a sense of being detached from one's self and one's emotions, as well as a perception of one's surroundings as unreal. Symptoms of dissociation most often are studied in relation to the sequelae of severe abuse or trauma (Schimmenti et al., 2012), and dissociation can serve as a coping strategy, as a means of escaping from the painful feelings elicited by experienced trauma. However, even in the absence of trauma individuals may experience mild dissociative symptoms that include brief gaps in awareness or lapses in memory, experiences of imaginative involvement and experiences of intense absorption (Cardena \& Carlson, 2011). Taking into consideration the specifics of internet use, which often includes identification with certain characters in a video game or the development of an "avatar" for use in social networking, hence absorption into a virtual world, it seems somewhat surprising 
that researchers of internet use have paid so little attention to possible associations with dissociation. Studies have shown that a high frequency of dissociative experience predicts various socioemotional problems, including difficulties in personal relationships, difficulties with functioning at school or work, as well as distress from nightmares (e.g. Secrist, Dalenberg, \& Grevitz, 2019).

The majority of studies examining the negative sequelae of internet use have been cross-sectional, with some exceptions. The question has been raised as to whether internet usage leads to mental health problems or do mental health problems lead young people to use the internet in problematic ways? Or is there a bidirectional, reciprocal relationship? A fairly recent four-year longitudinal study on adolescent CIU and mental health showed that CIU predicted the development of poor mental health, but that poor mental health did not predict CIU development (Ciarrochi et al., 2016). However, the authors of this study indicate that the General Health Questionnaire used in their study may have been too broad to pick up the consequences of poor mental health. The purpose of the present study was to continue addressing this question of directionality of the relationships between CIU and aspects of mental health difficulty, in this case, examining specifically the associations with depression, anxiety and dissociation, with measurements at two points in time.

The research questions posed were as follows: What are the associations between adolescent CIU and symptoms of depression, anxiety and dissociation at Time 1 and Time 2? Is there a mediating effect of dissociation between CIU at Time 1 and Time 2?

\section{Methodology}

\section{Participants}

Participating in the study were 80 adolescents (39 girls and 41 boys), ages $12-18$ years old (mean age 14.90 years, SD 2.02) at Time 1 . The adolescents participating in the study were from two different schools in Latvia, chosen according to accessibility. The majority of the study participants (73\%) reported that they are living with both parents, while $19 \%$ reported living in a singleparent household with their mother, and 8\% reported other living arrangements, such as living with their grandparents. Almost all of the study participants reported that computers and smart phones are readily available to them: $98 \%$ reported that they have a computer at home; $96 \%$ reported that they have internet access at home; $94 \%$ reported that they have their own smart phone with internet access. 


\section{Procedure}

Permission was received from the school principals to conduct the study. Parents were informed of the content of the study with information posted on the online platform for teacher-parent communication, and passive consent was received, meaning that the parents were advised to indicate if they did not want their child to participate. None of the parents indicated that they did not want their child to participate. Students were informed about the purpose of the study, and principles of anonymity, voluntary participation and the right to withdraw from the study at any time without repercussions were explained. All of the adolescents who were invited to participate in the study agreed to participate with active consent. Questionnaires were distributed and collected by trained research assistants during homeroom class sessions. The homeroom teachers were not involved with the data collection process. Data was collected at Time 1, and again one year later at Time 2.

\section{Measures}

All measures were independently forward and back-translated from English to Latvian by several bilingual translators. Consensus agreement was reached in case of any discrepancies. The translations were pilot-tested and after initial psychometric analysis, the translations of some items were refined in order to ensure conceptual equivalence of the items in comparison to the original.

Compulsive internet use was assessed with four items from the Compulsive Internet Use Scale (Meerkerk et al., 2009). Adolescents reported on items about preoccupation with using the internet; the need to use increased amounts of time on the internet; and feeling restless, moody or depressed when not using the internet. Adolescents rated the items on a 5-point scale, ranging from 1 (never) to 5 (always). Internal consistency for the four-item scale was moderate in our sample: Cronbach's alpha ranged from 0,73 to 0,83 at Time 1 and Time 2

Depression, anxiety and dissociation symptoms were self-reported by adolescents with use of the Trauma Symptom Checklist (Briere, 1995). Adolescents rated the items on a 5-point Likert-type scale from 1 (never) to 5 (always). This study included analysis of three scales: depression, anxiety and dissociation. The depression scale includes 9 items (e.g., I feel sad or unhappy; I feel that nobody likes me); the anxiety scale includes 9 items (e.g., I feel afraid that something bad might happen); and the dissociation scale includes 9 items (e.g. I pretend that I am someone else; I feel that things aren't real). In our sample internal consistency was good for all three scales at Time 1 and Time 2: Cronbach's alpha for depression was at 0,86 and 0,87; Cronbach's alpha for anxiety was at 0,80 and 0,83 ; and Cronbach's alpha for dissociation scale was at .87 at both measurement times. 
SOCIETY. INTEGRATION. EDUCATION

Proceedings of the International Scientific Conference. Volume VII, May $22^{\text {th }}-23^{\text {th }}, 2020.136-144$

Adolescents also reported their age, gender and availability of internet use.

\section{Data Analysis}

First, preliminary descriptive analyses, and zero-order correlational analyses were conducted to examine bivariate associations among the adolescents' ratings of depression, anxiety and dissociation symptoms and PIU. The possibility of a mediation effect was tested with a series of four regression analyses: 1) dissociation was regressed on CIU at Time 1;2) CIU at Time 2 was regressed on dissociation; 3) CIU at Time 2 was regressed on CIU at Time 1; and 4) CIU at Time 2 was regressed on dissociation and CIU at Time 1 . The Sobel test was conducted to examine the mediation effect.

\section{Research results}

Descriptive statistics and bivariate associations (Spearman correlations) between the study variables are presented in Table 1.

Table 1 Descriptive Statistics and Correlations between CIU, Depression, Anxiety and Dissociation at Time 1 and Time 2

\begin{tabular}{|l|c|c|c|c|c|c|c|c|}
\hline & $\mathbf{1 .}$ & $\mathbf{2 .}$ & $\mathbf{3 .}$ & $\mathbf{4 .}$ & $\mathbf{5 .}$ & $\mathbf{6 .}$ & $\mathbf{7 .}$ & $\mathbf{8 .}$ \\
\hline 1. CIU 1 & & $0,56^{* *}$ & $0,32^{* *}$ & $0,31^{* *}$ & $0,40^{* *}$ & $0,36^{* *}$ & $0,56^{* *}$ & $0,49^{* *}$ \\
\hline 2. CIU 2 & & & 0,15 & $0,36^{* *}$ & 0,20 & $0,43^{* *}$ & $0,27^{*}$ & $0,42^{* *}$ \\
\hline 3. Depression 1 & & & & $0,58^{* *}$ & $0,73^{* *}$ & $0,46^{* *}$ & $0,68^{* *}$ & $0,47^{* *}$ \\
\hline 4. Depression 2 & & & & & $0,45^{* *}$ & $0,77^{* *}$ & $0,44^{* *}$ & $0,74^{* *}$ \\
\hline 5. Anxiety 1 & & & & & & $0,55^{* *}$ & $0,69^{* *}$ & $0,44^{* *}$ \\
\hline 6. Anxiety 2 & & & & & & & $0,49^{* *}$ & $0,74^{* *}$ \\
\hline 7.Dissociation 1 & & & & & & & & $0,52^{* *}$ \\
\hline 8.Dissociation 2 & & & & & & & & \\
\hline M & 2,35 & 2,35 & 3,10 & 1,94 & 2,09 & 1,98 & 2,03 & 1,94 \\
\hline$S D$ & 0,88 & 0,76 & 0,72 & 0,72 & 0,66 & 0,62 & 0,72 & 0,70 \\
\hline
\end{tabular}

Note: ${ }^{*} p<0,05,{ }^{* *} p<0,01$. CIU $1=$ Compulsive Internet Use at Time 1; CIU $2=$ Compulsive Internet Use at Time 2. $n=80$

CIU at Time 1 was found to be positively correlated with ratings of depression at Time $1(\mathrm{r}=0,32, \mathrm{p}<0,01)$ and Time $2(\mathrm{r}=0,31, \mathrm{p}<0,01)$; with ratings of anxiety at Time $1(\mathrm{r}=0,40, \mathrm{p}<0,01)$ and Time $2(\mathrm{r}=0,36, \mathrm{p}<0,01)$; and with ratings of dissociation at Time $1(\mathrm{r}=0,56, \mathrm{p}<0,01)$ and Time 2 $(r=0,49, p<0,01)$. CIU at Time 2 was found to be positively correlated with 
ratings of depression at Time $2(r=0,36, p<0,01)$; with ratings of anxiety at Time $2(\mathrm{r}=0,43, \mathrm{p}<0,01)$; and with ratings of dissociation at Time $1(\mathrm{r}=0,27$, $\mathrm{p}<0,05)$ and Time $2(\mathrm{r}=0,42, \mathrm{p}<0,01)$. Also examined were possible correlation between the above mentioned variables with age and gender. The only significant associations that was found was between gender and depression at Time $1(\mathrm{r}=0,25, \mathrm{p}<0,05)$; gender and depression at Time 2 was with a tendency towards significance $(r=-0,21, p=0,057$, respectively), indicating that girls reported higher depression ratings than boys. Correlation analysis also showed that the time spent using the internet was positively associated with CIU.

An examination of mediation effects showed dissociation at Time 2 to mediate the association between hyperactivity and maladaptive cognitions (see Table 2). Initial results showed a significant direct relation between CIU at Time 1 (CIU 1 ) and CIU at Time $2(\beta=0,58, S E=0,08)$, as well as a significant direct relation between CIU 1 and Dissociation at Time $2(\beta=0,53, S E=0,08)$. Upon entering both CIU 1 and Dissociation at Time 2 in prediction of CIU 2, the change in predictive value $(\beta=0,48, S E=0,09)$ indicated a partial mediating effect of Dissociation.

Table 2 Dissociation mediating the association between CIU at Time 1 and Time 2

\begin{tabular}{|c|c|c|c|c|c|c|}
\hline & & B & $S D(B)$ & $\beta$ & $F$ & $R^{2}$ \\
\hline 1. & $\begin{array}{c}\longrightarrow \\
\text { Dissociation } 2\end{array}$ & 0,42 & 0,08 & $0,53 * * *$ & $29,86^{* * *}$ & 0,28 \\
\hline 2. & $\begin{array}{ll}\text { Dissociation 2 } & \longrightarrow \\
& \text { CIU 2 }\end{array}$ & 0,48 & 0,11 & $0,44 * * *$ & $19,34 * * *$ & 0,20 \\
\hline 3. & $\begin{array}{ll}\text { CIU } 1 \longrightarrow \\
\end{array}$ & 0,50 & 0,08 & $0,58 * * *$ & $39,08 * * *$ & 0,33 \\
\hline 4. & $\begin{array}{r}\text { Predicting CIU } 2 \\
\text { CIU } 1 \\
\text { Dissociation } 2\end{array}$ & $\begin{array}{l}0,42 \\
0,20\end{array}$ & $\begin{array}{l}0,09 \\
0,12\end{array}$ & $\begin{array}{l}0,48 * * \\
0,18\end{array}$ & $21,43 * * *$ & 0,36 \\
\hline
\end{tabular}

Note: ${ }^{* *} p<0,01 ; * * * p<0,001$. CIU 1 = Compulsive Internet Use at Time 1; CIU $2=$ Compulsive Internet Use at Time 2; Dissociation $2=$ Dissociation at Time 2. $n=80$

The Sobel test confirmed the partial mediation effect, $\mathrm{z}=3,36, \mathrm{p}<0,01$. 


\section{Discussion and Conclusions}

The aim of this study was to examine adolescent CIU across a one-year time period in association with symptoms of depression, anxiety and dissociation, as well as to analyze the potential mediating effect of dissociation symptoms. The initial correlational analysis showed that CIU at Time 1 (CIU 1) is strongly associated with CIU at Time 2 (CIU 2). Furthermore, CIU 1 is associated with depression, anxiety and dissociation variables - both as reported at Time 1 and Time 2. These results are in support of previous cross-sectional studies which have shown CIU to be associated with symptoms of anxiety and depression (e.g. Carli et al., 2012). These results also support the study by Ciarocchi and colleagues (2016), which showed mental health problems to be the sequelae of problematic internet use, rather than a contributing risk factor. In fact, also in this study Depression at Time 1 was not associated with CIU 2, and neither was Anxiety at Time 1 associated with CIU 2, thereby indicating that depression and anxiety do not have a causal relationship in facilitating CIU. Of course, the results of this study need to be replicated with a larger sample and across a longer period of time.

The associations between CIU and dissociation symptoms indicate a somewhat different pattern. The adolescent ratings of dissociation at both Time 1 and Time 2 were associated with CIU 2, providing indication that dissociation symptoms may be both a facilitating factor and also a consequence of problematic internet use, which would entail a bidirectional effect of causality. Such a stipulation would be in line with previous studies which have shown that vulnerable individuals can be progressively drawn into the virtual world of the internet (Liu \& Peng, 2009). Adolescents with symptoms of dissociation, which by definition include a tendency to become absorbed with various mediums that provide escape from reality (including books and films), may be especially vulnerable to the enticements of the virtual world (Schimmenti et al., 2012). The results from this study showed the effect of partial mediation by dissociation symptoms between CIU 1 and CIU 2, again indicating that adolescents who are prone to dissociation and who use dissociation as a coping strategy would be particularly susceptible to being drawn into the virtual world of the internet, and this could contribute to overinvolvement with either video gaming or social networking.

As indicated above, among the limitations of the present study is the relatively small sample size, which should be expanded in future studies concerning CIU across time. Also expanded in future studies should be the length of the time span studied longitudinally. It would be valuable to include at least three measurement times, so as to more fully understand the relationship between symptoms of psychopathology as potential precursors to problematic 
internet use, as well as the symptoms as sequelae of CIU. Potentially there could also be bidirectional effects, as implied by the results of the current study in the found associations between CIU at Time 1 and Time 2, and dissociation symptoms at Time 1 and Time 2. Future studies should also include a measure of early experiences of neglect and abuse, or other forms of trauma, which have previously been shown to contribute to dissociative processes (Schimmenti et al., 2012).

The practical implications of this study accentuate the need for parents and educators to be aware of the costs and benefits of extended internet use, and to seek the most appropriate and effective means by which to limit extensive internet use that can lead to problematic internet use, symptoms of withdrawal, preoccupation and subsequent difficulties in social relationships and academic schoolwork. Furthermore, the results from this study indicate that particular attention should be paid to the possibility of extensive internet use facilitating over-involvement and detachment from reality as the internet potentially provides psychological absorption in an alternative reality, but away from the benefits which everyday reality can and should provide.

\section{References}

Briere, J. (1995). Trauma Symptom Checklist for Children: Professional manual. Odessa, FL: Psychological Assessment Resources.

Cardena, E.B., \& Carlson, E.B. (2011). Acute Stress Disorder revisited. Annual Review of Clinical Psychology, 7, 245-267.

Carli, V., Durkee, T., Wasserman, D., Hadlaczky, G., Despalins, R., Kramarz, E., ... Kaess, M. (2012). The association between pathological internet use and comorbid psychopathology: A systematic review. Psychopathology, 46, 1-13.

Ciarrochi, J., Parker, P., Sahdra, B., Marshall, S., Jackson, C., Gloster, A.T., \& Heaven, P. (2016). The Development of compulsive internet use and mental health: A four-year study of adolescence. Developmental Psychology, 52(2), 272-283.

Dalbudak, E., Evren, C., Aldemir, S., \& Evren, B. (2014). The severity of internet addiction risk and its relationship with the severity of borderline personality features, childhood traumas, dissociative experiences, depression and anxiety symptoms among Turkish university students. Psychiatry Research, 219, 577-582.

De Berardis, D., D'Albenzio, A., Gambi, F., Sepede, G., Valchera, A., Conti, C.M., \& Ferro, F.M. (2009). Alexithymia and its relationships with dissociative experiences and Internet addiction in a nonclinical sample. CyberPsychology and Behavior, 12, 67-69.

Ekbia, H., \& Nardi, B. (2012). Inverse instrumentality: How technologies objectify patients and players. In P. Leonardi, B. Nardi, \& J. Kallinikos (Eds.), Materiality and Organizing: Social Interaction in a Technological World (pp. 157-176). Oxford: Oxford University Press.

Kaess, M., Durkee, T., Brunner, R., Carli, V., Parzer, P., Wasserman, C., ... Wasserman, D. (2014). Pathological internet use among European adolescents: Psychopathology and self-destructive behaviours. European Child \& Adolescent Psychiatry, 23, 1093-1102. 
Liu, M., \& Peng, W. (2009). Cognitive and psychological predictors of the negative outcomes associated with playing MMOGs. Computer in Human Behavior, 25, 1306 -1311.

Mazzoni, E., Cannata, D., \& Baiocco, L. (2017). Focused, not lost: The mediating role of Temporal Dissociation and Focused Immersion on Problematic Internet Use. Behaviour \& Information Technology, 36(1), 11-20.

Meerkerk, G.-J., Van Den Eijnden, R.J.J.M., Vermulst, A.A., \& Garretsen, H.F.L. (2009). The Compulsive Internet Use Scale (CIUS): Some psychometric properties. Cyber Psychology and Behavior, 12(1), 1-6

Probst, T., Berger, T., Meyer, B., Späth, C., Schröder, J., Hohagen, F., Moritz, S., \& Klein, J.P. (2020). Social phobia moderates the outcome in the EVIDENT study:

A randomized controlled trial on an internet-based psychological intervention for mild to moderate depressive symptoms. Journal of Consulting and Clinical Psychology, 88(1), 82-89.

Schimmenti, A., Guglielmucci, F., Barbasio, C., \& Granieri, A. (2012). Attachment disorganization and dissociation in virtual worlds: A study on problematic Internet use among players of online role playing games. Clinical Neuropsychiatry: Journal of Treatment Evaluation, 9(5), 195-202.

Secrist, M.E., Dalenberg, C.J., \& Gevirtz, R. (2019). Contributing factors predicting nightmares in children: trauma, anxiety, dissociation, and emotion regulation. Psychological Trauma: Theory, Research, Practice, and Policy, 11(1), 114-121.

Young, K.S. (1998). Internet addiction: The emergence of a new clinical disorder. Cyber Psychology and Behavior, 1, 237-244. 